\title{
Comportement thermique de films minces de verres $\mathrm{GeSe}_{y}$
}

\author{
Michèle Dubourg $\left({ }^{1}\right)$, Bernard Cros $\left({ }^{2}\right)$, Michel Ribes $\left({ }^{2}\right)$, Jean-Paul Martinez $\left({ }^{1}\right)$ \\ et Jean-Louis Balladore $\left({ }^{1}\right)$ \\ ( $\left.{ }^{1}\right)$ Centre d'Elaboration de Matériaux et d'Etudes Structurales, Laboratoire d'Optique \\ Electronique - CNRS, 29 rue Jeanne Marvig, 31055 Toulouse Cedex, France \\ $\left({ }^{2}\right)$ Laboratoire de Physicochimie des Matériaux Solides, USTL, place E. Bataillon, \\ 34095 Montpellier Cedex 5, France
}

(Reçu le 23 mai 1990, accepté le 14 décembre 1990)

Résumé. - Des films minces de verres de chalcogenure $\mathrm{GeSe}_{y}$ ont été préparés par PECVD en vue d'applications pour la lithographie. Leur morphologie à différentes températures est observée par microscopie électronique. Leur composition locale est évaluée par analyse X.

\begin{abstract}
Thin films of chalcogenide glasses $\mathrm{GeSe}_{y}$ were prepared by PECVD for lithographic application. Their morphology at different temperatures is observed by electron microscopy. Their local composition is evaluated by $\mathrm{X}$ analysis.
\end{abstract}

\section{Introduction.}

Les verres de chalcogénure sensibilisés a l'argent et plus particulièrement les verres $\mathrm{GeSe}_{y}$, préparés sous forme de films minces, font l'objet de nombreuses études en raison de leur application comme couches photosensibles en microlithographie $[1,2]$.

Nous avons étudié antérieurement la morphologie de couches minces $\mathrm{GeSe}_{y}$ déposées par PECVD (Plasma Enhanced Chemical Vapor Deposition), technique bien adaptée à la réalisation à basse température de dépôts sur des surfaces importantes, avec des rendements élevés. Une structure démixtée en microdomaines dont la taille évolue avec la composition globale du verre a été mise en évidence [3]. La présente étude a pour but de contrôler la stabilité thermique de ce type de morphologie.

\section{Méthodes expérimentales.}

Les couches, de composition $\mathrm{GeSe}_{3}$ et $\mathrm{GeSe}_{5,5}$ ont été déposées par PECVD, selon un procédé décrit antérieurement [4] sur des substrats de silicium de diamètre 4 pouces, recouverts d'une couche de polymère (résine OFP). Les films, d'épaisseur $70 \mathrm{~nm}$, sont séparés du substrat par 
dissolution du polymère dans un bain d'acétone, puis recueillis sur des grilles de platine de $3 \mathrm{~mm}$ (400 mesh).

Les observations sont effectuées à l'aide d'un microscope Philips EM400, 120 keV. Il est équipé d'un filament $\mathrm{LaB}_{6}$, ce qui permet d'obtenir dans certaines conditions, une sonde de $10 \mathrm{~nm}$ de diamètre. L'appareil est couplé à un spectromètre X TRACOR à dispersion d'énergie qui permet d'analyser quantitativement la composition chimique de matériaux constitués d'éléments de numéro atomique élevé $(Z>13)$. Pour la détection d'éléments plus légers le microscope est équipé d'un spectroscope de perte d'énergie des électrons transmis de type VG (Vacuum Generator) à détection séquentielle. Pour cette étude, l'échantillon est placé sur un porte-objet chauffant dont la température est pilotée par un programme. Le miscroscope est également équipé de bobines déflectrices situées au-dessus de la lentille condenseur et permettant de dévier le faisceau entre deux observations lors d'une variation de la température de l'échantillon afin de ne pas irradier ce dernier.

\section{Résultats.}

3.1 FILM DE COMPOSITION GeSe 3 . - A la température ambiante, l'image en champ clair est celle d'une couche uniforme dont le diagramme de diffraction est composé de quatre anneaux diffus présentés sur la figure 1.

Tableau I. - Distances mesurées à partir du diagramme de diffraction.

[Distances measured from the diffraction diagram.]

\begin{tabular}{|cc|}
\hline Anneau & $d(\mathrm{~nm})$ \\
1 & 0,595 \\
2 & 0,296 \\
3 & 0,177 \\
4 & 0,112 \\
\hline
\end{tabular}

Les distances correspondantes à ces anneaux sont données dans le tableau I ; elles correspondent à celles relevées sur le même type de matériau [4].

L'analyse X confirme la composition nominale $\mathrm{GeSe}_{3}$ (Fig.2). (Les pics du platine et du cuivre sont dus à la grille-support et au porte-objet. Tous les pics sont mesurés en unité arbitraire).

Le calcul des concentrations atomiques en Ge et Se est effectué à partir des raies K. Le logiciel d'analyse TRACOR utilisé prend en compte les convolutions entre les différents pics. Dans ces conditions la précision des mesures peut être évaluée à $10 \%$.

Le chauffage de l'échantillon étant programmé à $+2^{\circ}$ par minute, une première modification est observée à $250^{\circ} \mathrm{C}$ : apparition de taches sombres dont la taille varie entre 100 et $400 \mathrm{~nm}$ (photo a, Fig.3). Une analyse $X$ de ces nodules ne permet pas de mettre en évidence une évolution de la composition. Par contre en diffraction on a apparition de quelques taches qui se superposent au diagramme précédent : ce phénomène peut s'interpréter comme l'amorce d'une cristallisation.

Entre $250^{\circ} \mathrm{C}$ et $300^{\circ} \mathrm{C}$ le nombre de germes augmente et on observe à partir de $300^{\circ} \mathrm{C}$ la croissance de cristaux (Fig.3b). Le diagramme de diffraction (Fig.3c) est caractéristique d'un mono- 


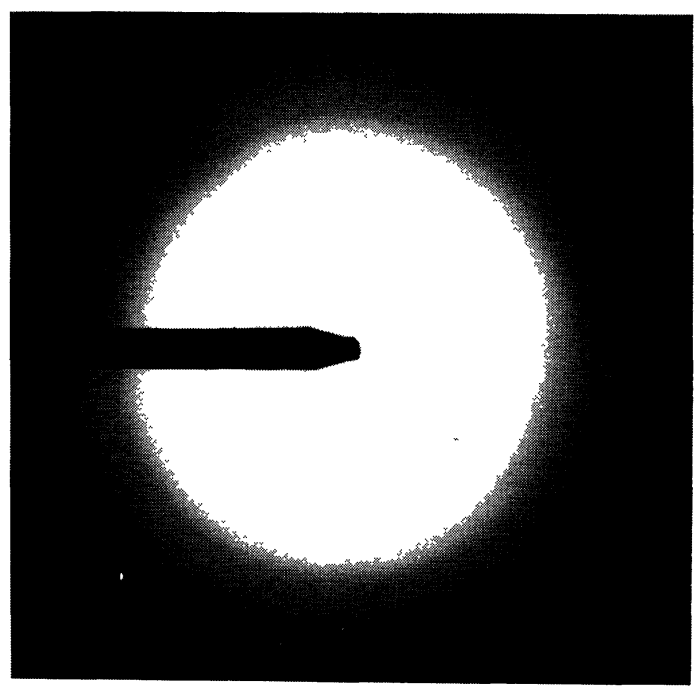

Fig. 1. - Diagramme de diffraction d'une couche $\mathrm{GeSe}_{3}$ à $25^{\circ} \mathrm{C}$ (seuls 3 anneaux sont nettement visibles sur la reproduction; le quatrième de très faible intensité est présent sur le négatif).

[Diffraction diagram of $\mathrm{GeSe}_{3}$ film at $25^{\circ} \mathrm{C}$ (only 3 rings are visible in the Fig. ; the 4th one has a to weak intensity but it is present on the negative film).]

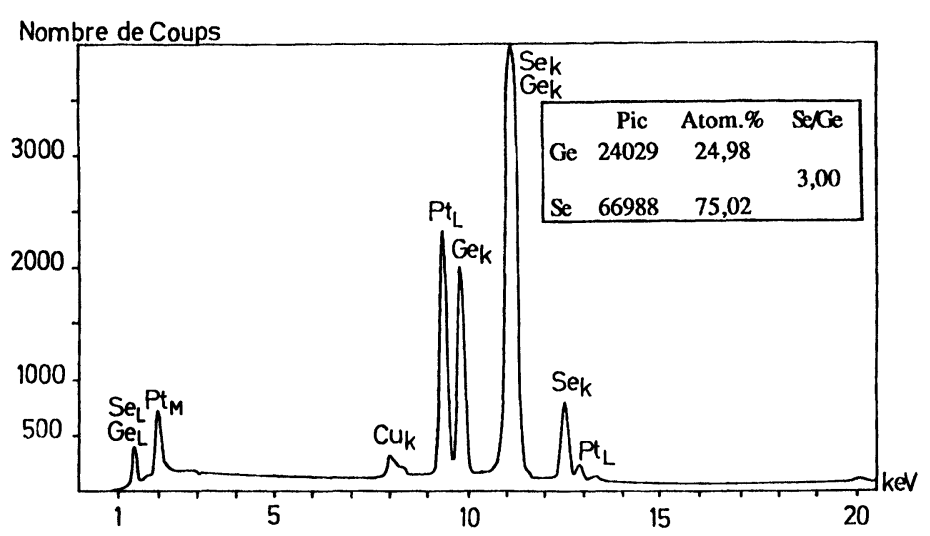

Fig. 2. - Spectre $\mathrm{X}$ du $\mathrm{GeSe}_{3}$ et concentration des différents éléments (les pics sont évalués en nombre de coups en fonction de l'energie en $\mathrm{keV}$ ).

[X-ray spectrum of $\mathrm{GeSe}_{3}$ and concentration of different elements (the peaks are evaluated in counts as a function of energy in $\mathrm{keV})$.]

cristal de $\mathrm{GeSe}_{2}$. L'indexation de ce diagramme confirme la structure orthorombique ( $a=0,44$ $\mathrm{nm}, b=1,082 \mathrm{~nm}, c=0,385 \mathrm{~nm}$ ) proposée par certains auteurs [5].

L'analyse de ces cristaux met en évidence une composition proche du $\mathrm{GeSe}_{2}$ (Tab.II) ce qui est en accord avec le fait que les verres $\mathrm{GeSe}_{y}$ cristallisent sous la seule forme $\mathrm{GeSe}_{2}[5,6]$.

A ce stade, dans une première expérience, le chauffage a été poursuivi. Lorsque l'échantillon 

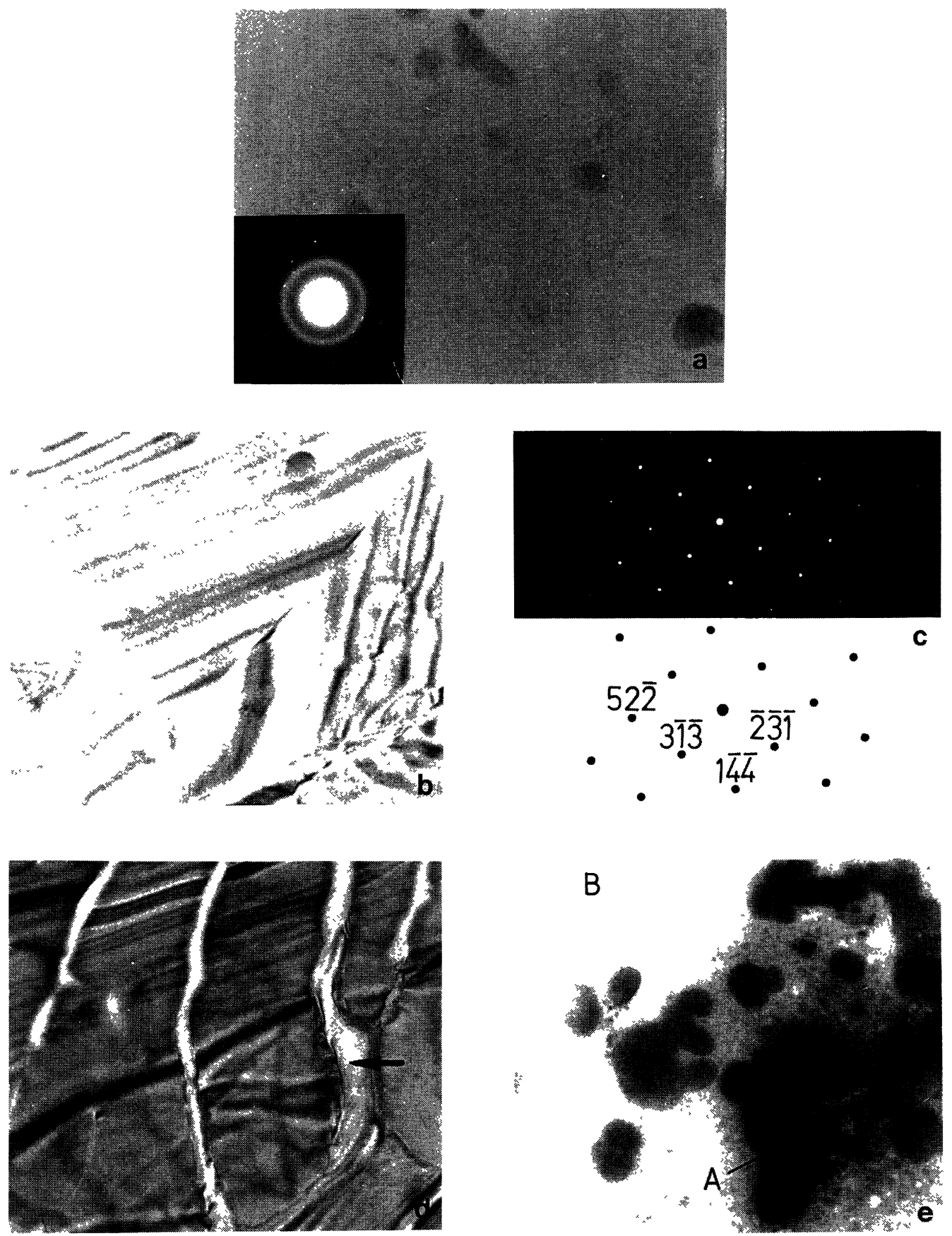

Fig. 3. - a) $\mathrm{GeSe}_{3}$ à $250^{\circ} \mathrm{C}$ (grandissement 19500) et diagramme de diffraction. b) Matériau cristallisé à $300^{\circ} \mathrm{C}$ (grandissement 33000 ). c) Diagramme de diffraction du matériau cristallisé sous forme $\mathrm{GeSe}_{2}$. d) Matériau à $330^{\circ} \mathrm{C}$ (grandissement 33000). e) Matériau à température ambiante après $48 \mathrm{~h}$. (grandissement 42000).

[a) $\mathrm{GeSe}_{3}$ at $250^{\circ} \mathrm{C}$ (magnification 19500) and diffraction diagram. b) Crystallized material at $300^{\circ} \mathrm{C}$ (magnification 33000). c) Diffraction diagram of $\mathrm{GeSe}_{2}$ glass. d) Material at $330^{\circ} \mathrm{C}$ (magnification 33000 ). e) Material at room temperature after $48 \mathrm{~h}$. (magnification 42000).] 
est à une température de $330^{\circ} \mathrm{C}$, l'image en champ clair montre que des zones amorphes se développent à partir des joints de grains (photo d, Fig.3). Ce phénomène est dû à une fusion du matériau. Une analyse $\mathrm{X}$ de ces régions (flèche) montre que l'on retrouve la composition $\mathrm{GeSe}_{3}$ de départ (Tab.III).

Au cours d'une seconde expérience, un abaissement de la température de $-2^{\circ} \mathrm{C}$ par minute a été imposé à partir de $300^{\circ} \mathrm{C}$. L'échantillon conserve, au cours du refroidissement, son état cristallisé jusqu'à la température ambiante. Laissé à l'air pendant 48 heures, à l'abri de la lumière, il évolue avec ségrégation des composants : la photo e, figure 3 donne un aspect de la préparation à la température ambiante. Les tableaux IV et V mettent en évidence les compositions différentes des zones A et B.

Une étude en régime statique de ces phénomènes a été entreprise : après avoir atteint une valeur donnée de la température on la maintient constante pendant 30 minutes en ayant soin de déflecter le faisceau du microscope. Les observations effectuées après ce laps de temps conduisent à des résultats tout à fait analogues à ceux décrits plus haut.

Tableau II. - Analyse X d'un monocristal à $300^{\circ} \mathrm{C}$.

[X-ray analysis of a monocrystal at $300^{\circ} \mathrm{C}$.]

\begin{tabular}{|cclc|}
\hline & Pic & Atom.\% & Se/Ge \\
Ge & 52789 & 31,08 & \\
& & & 2,22 \\
Se & 108630 & 68,92 & \\
\hline
\end{tabular}

Tableau III. - Analyse $X \grave{a ̀ ~} 330^{\circ} \mathrm{C}$.

[X-ray analysis at $330^{\circ} \mathrm{C}$.]

\begin{tabular}{|cccc|}
\hline & Pic & Atom\% & Se/Ge \\
Ge & 8934 & 24,2 & \\
& & & 3,13 \\
Se & 25962 & 75,79 & \\
\hline
\end{tabular}

Tableau IV. - Analyse zone A, figure $3 e$.

[X-ray analysis of part A, figure $3 \mathrm{e}$.

\begin{tabular}{|cccc|}
\hline & Pic & Atom $\%$ & Se/Ge \\
Ge & 321 & 13,73 & \\
& & & 6,28 \\
Se & 1870 & 86,18 & \\
\hline
\end{tabular}

Tableau V. - Analyse zone B, figure $3 e$.

[X-ray analysis of part $\mathrm{B}$, figure $3 \mathrm{e}$.]

\begin{tabular}{|cccc|}
\hline & Pic & Atom $\%$ & Se/Ge \\
Ge & 7445 & 77,32 & \\
& & & 0,29 \\
Se & 2025 & 22,67 & \\
\hline
\end{tabular}


3.2 FILM GeSe s,5 $_{\text {. }}$ - Le même protocole de manipulation est appliqué au film de composition $\mathrm{GeSe}_{5,5}$. Le résultat de l'analyse $\mathrm{X}$ à température initiale (Fig.4) confirme une composition voisine de celle prévue lors de l'élaboration.

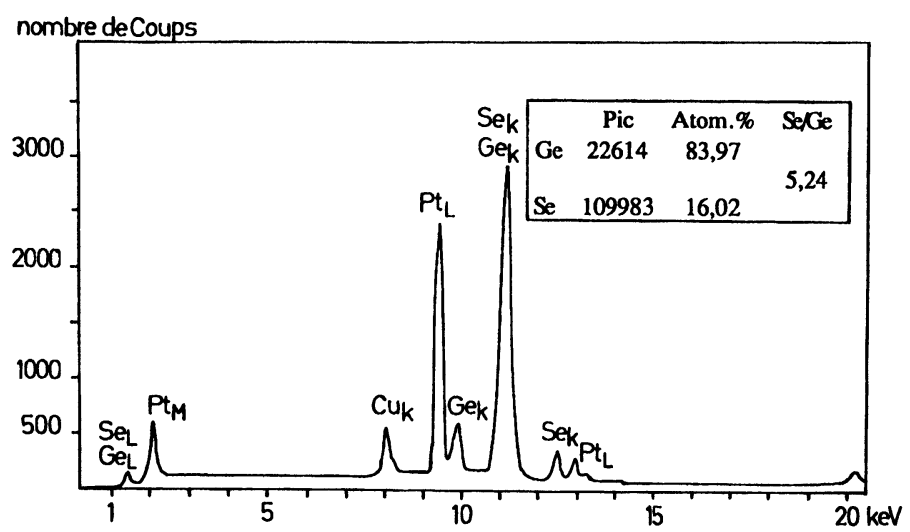

Fig. 4. - Analyse $\mathrm{X}$ du film $\mathrm{GeSe}_{5,5}$.

[X-ray analysis of $\mathrm{GeSe}_{5.5}$ film.]

Dans l'ensemble le comportement thermique est semblable à celui du film $\mathrm{GeSe}_{3}$ : l'élévation de la température conduit à une phase cristalline. Cependant une modification de la morphologie se produit dès $70^{\circ} \mathrm{C}$ (photo a, Fig.5) : l'image en champ clair montre des domaines plus contrastés dont les tailles s'échelonnent de 50 à $200 \mathrm{~nm}$. Ils peuvent être considérés comme les premiers germes de cristallisation : un diagramme de points se superpose au diagramme du verre.

A $300^{\circ} \mathrm{C}$ les cristaux se développent (photo b, Fig.5). La cristallisation n'est totale qu'à $330^{\circ} \mathrm{C}$ (photo c, Fig 5). Deux phases cristallines apparaissent. Dans la plus grande partie (zone A) l'analyse X (Tab. VI) et le diagramme de diffraction indiquent la présence de $\mathrm{GeSe}_{2}$ cristallisé. La zone B est constituée d'ilôts de sélénium cristallisé (Tab. VII). Le diagramme de diffraction correspondant (photo 2d, Fig. 5) montre une structure hexagonale de paramètres : $a=43,662 \mathrm{~nm}$, $c=49,536 \mathrm{~nm}$. Cette ségrégation qui n'était pas observée pour le $\mathrm{GeSe}_{3}$ peut être attribuée à la composition de départ $\left(\mathrm{GeSe}_{5,5}\right)$ plus riche en sélénium.

Tableau VI. - Analyse zone A, photo $5 \mathrm{c}$.

[X-ray analysis of part A, figure 5c.]

\begin{tabular}{|cccc|}
\hline & Pic & Atom $\%$ & Se/Ge \\
Ge & 16962 & 30,73 & \\
& & & 2,25 \\
$\mathrm{Se}$ & 35489 & 69,27 & \\
\hline
\end{tabular}



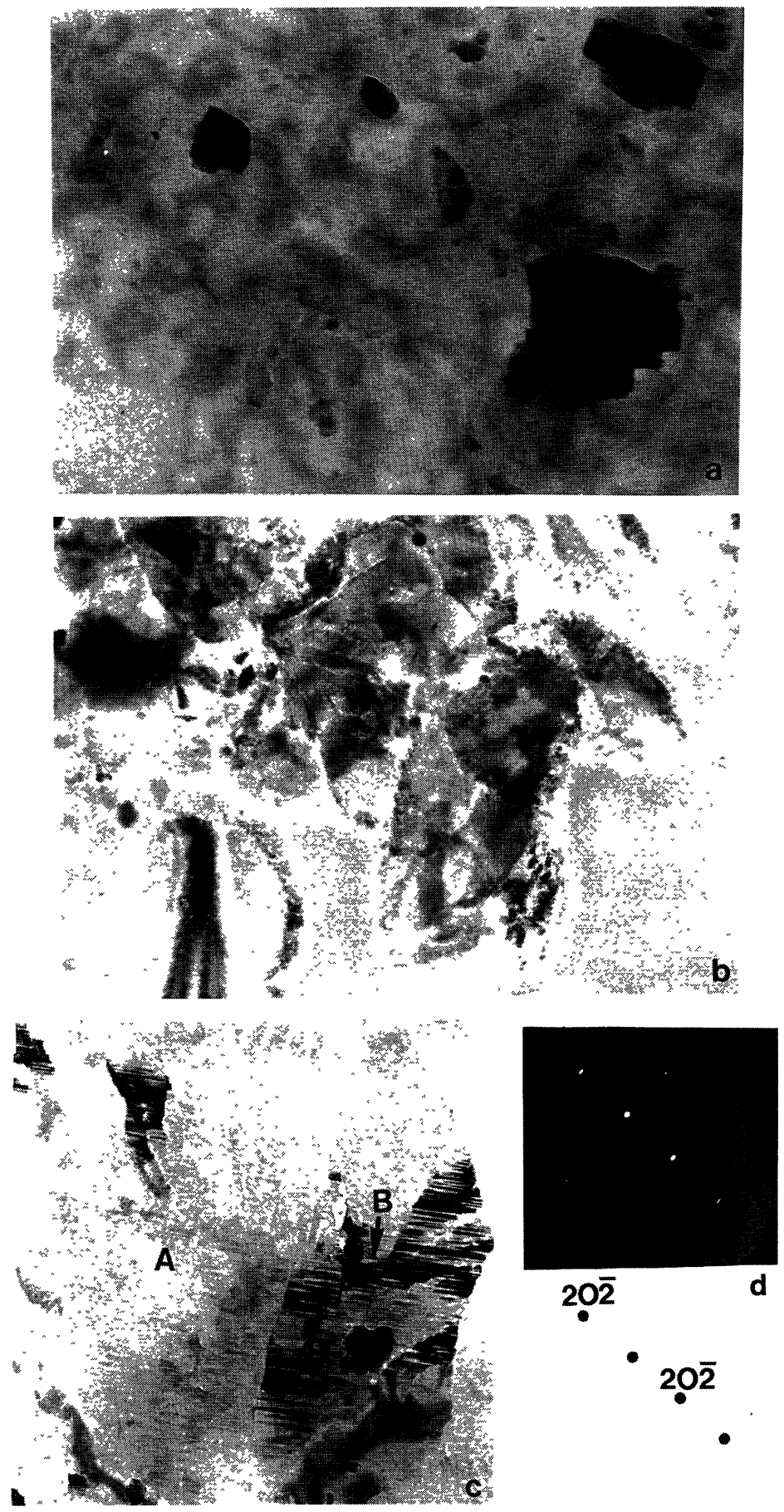

Fig. 5. - a) $\mathrm{GeSe}_{5,5} 5^{\circ} 70^{\circ} \mathrm{C}$ (grandissement 33000). b) Matériau à $300^{\circ} \mathrm{C}$ (grandissement 33000). c) Matériau à $330^{\circ} \mathrm{C}$. ségrégation du sélénium en ilôts (grandissement 33000). d) Diagramme de diffraction d'un ilôt de sélénium cristallisé (zone B).

[a) $\mathrm{GeSe}_{5,5}$ at $70^{\circ} \mathrm{C}$ (magnification 33000 ). b) Material at $300^{\circ} \mathrm{C}$ (magnification 33000). c) Material at $330^{\circ} \mathrm{C}$. Selenium segregation (magnification 33000). d) Diffraction diagram of the crystallized selenium (part B).] 
Tableau VII. - Analyse zone B, photo $5 c$.

[X-ray analysis of part B, figure $5 \mathrm{c}$.]

\begin{tabular}{|cccc|}
\hline & Pic & Atom $\%$ & Se $/ \mathrm{Ge}$ \\
Ge & 0 & 0,0 & \\
Se & 2025 & 99,98 & 9998 \\
\hline
\end{tabular}

\section{Conclusion.}

Nous avons observé le comportement thermique des verres $\mathrm{GeSe}_{y}$. Une élévation de la température conduit, quelle que soit la composition, à une cristallisation.

Cependant, suivant la richesse du matériau en sélénium, les températures de transition amorphe cristal varient.

Pour $y=3$ les premiers germes de cristallisation apparaissent à $250^{\circ} \mathrm{C}$ et à $300^{\circ} \mathrm{C}$ la totalité du matériau cristallise sous forme de $\mathrm{GeSe}_{2}$, l'excédent de sélénium semblant se localiser dans les joints de grains.

Pour $y=5,5$ les premières traces de cristallisation sont visibles à $70^{\circ} \mathrm{C}$. A $330^{\circ} \mathrm{C}$ l'ensemble du matériau est cristallisé sous deux formes : en $\mathrm{GeSe}_{2}$ comme dans le cas précédent et en ilôts de sélénium pur.

Utilisés comme couches photo ou electro sensibles dans une chaîne de fabrication de circuits intégrés, ces matériaux vont être soumis à des élévations de températures inférieures à $300^{\circ} \mathrm{C}$. Cela nécessite une étude plus précise de leur comportement au cours d'un chauffage prolongé (recuit).

\section{Bibliographie}

[1] Chen A.S., Addiego G., Leung W. et Neureuther A.R., J. Vac. Sc. Technol. B4 (1986) 398.

[2] Konan K., Galibert G. et Cala J., Phys. Stat. Sol. (a) 107 (1988) 273.

[3] Cros B., Camon H., Brocheton Y., Gonchond J.P., Tissier A., Balladore J.L. et Ribes M., J. Phys. France 50 (1989) C5-50.

[4] RiBES M., CROS B. et JULIEN P., Optical Microlithographic Technology for Integrated Circuit Fabrication and Inspection (SPIE-STOUER ed.) (1987) 811, 202.

[5] ElKORASHY A.M., Phys. Stat. Sol. (b) 135 (1986) 707.

[6] Phillips J.C., J. Non-Cryst. Sol. 34 (1979) 153. 